\title{
Validation of Cell-Free DNA Collection Tubes for Determination of EGFR Mutation Status in Liquid Biopsy from NSCLC Patients
}

\author{
Marta Sesé · Rosa Somoza - Inmaculada Maestu • Maria Martín Ureste • \\ Alfredo Sanchez · Juan Felipe Cordoba · Irene Sansano • \\ Griselda Venturas · Santiago Ramón y Cajal · Javier Hernández-Losa (iD
}

Received: May 31, 2019 / Published online: August 19, 2019

(C) The Author(s) 2019

\begin{abstract}
Introduction: Precision medicine has revolutionized the understanding and treatment of cancer by identifying subsets of patients who are amenable to specific treatments according to their molecular characteristics, as exemplified by epidermal growth factor receptor (EGFR) mutations in non-small cell lung cancer (NSCLC). Although tissue biopsy is the gold standard for determining molecular alterations in tumors, its limitations have prompted the
\end{abstract}

Enhanced Digital Features To view enhanced digital features for this article, go to https://doi.org/10.6084/ m9.figshare.9213077.

Electronic Supplementary Material The online version of this article (https://doi.org/10.1007/s40487019-00099-9) contains supplementary material, which is available to authorized users.

M. Sesé $\cdot$ R. Somoza $\cdot$ S. Ramón y Cajal .

J. Hernández-Losa $(\square)$

Translational Molecular Pathology, Vall d'Hebron Institute of Research (VHIR), Universitat Autònoma de Barcelona, Barcelona, Spain

e-mail: jahernan@vhebron.net

I. Maestu · M. M. Ureste

Department of Medical Oncology, Hospital

Universitario Doctor Peset, Valencia, Spain

A. Sanchez

Department of Medical Oncology, Consorci

Hospitalari Provincial de Castelló, Castellón, Spain development of new techniques for studying tumor biomarkers in liquid biopsies, such as mutation analysis in cell-free DNA (cfDNA). cfDNA analysis can accurately determine tumor progression and prognosis and more effectively identify appropriate targeted therapies. However, cfDNA is vulnerable, particularly during plasma sample shipping.

Objective: We compared the cell- and DNAstabilizing properties of cell-free DNA blood collection tubes (BCTs) with those of the traditional shipping method (frozen plasma) for EGFR mutation testing using the cobas ${ }^{\circledR}$ EGFR Mutation Test v2 in a prospective cohort of 49 patients from three different Spanish hospitals. Methods: In total, 98 NSCLC samples, two from each patient, were studied; five of the 49 cases were considered invalid by cobas ${ }^{\circledR}$ with one of the two shipping methods analyzed. After excluding these samples, we analyzed 88 samples from 44 patients. Considering the current

\author{
J. F. Cordoba \\ Department of Medical Oncology, Hospital \\ Universitari Arnau de Vilanova, Lleida, Spain \\ I. Sansano · G. Venturas · S. Ramón y Cajal · \\ J. Hernández-Losa \\ Department of Pathology, Hospital Universitari Vall \\ d'Hebron, Barcelona, Spain \\ M. Sesé · S. Ramón y Cajal · J. Hernández-Losa \\ Spanish Biomedical Research Network Centre in \\ Oncology (CIBERONC), Barcelona, Spain
}


methodology (frozen plasma) for sending samples as the gold standard, we evaluated the sensitivity and specificity of cfDNA BCT shipment.

Results: The global agreement between the two methods was $95.4 \%$, with $100 \%$ sensitivity and 94.6\% specificity for the cfDNA BCTs. cfDNA BCTs had a positive predictive value of $81.8 \%$ and negative predictive value of $100 \%$.

Conclusion: cfDNA BCTs have the same sensitivity for EGFR mutation analysis in liquid biopsy as the current methodology and very high specificity. They also have some additional advantages in terms of collection and further shipment. Therefore, cfDNA BCTs can be perfectly incorporated into the routine practice for EGFR mutation determination.

Funding: Roche Farma S.A., Spain.

Keywords: Blood collection tubes; cfDNA; EGFR mutation analysis; NSCLC

\section{INTRODUCTION}

Precision medicine has revolutionized the understanding and treatment of cancer by identifying subsets of patients who, according to their molecular characteristics, are amenable to specific treatments. Examples of predictive biomarkers of treatment outcomes include mutations in RAS and BRAF in colorectal cancer, BRAF in melanoma, c-KIT and PDGFR $\alpha$ in gastrointestinal stromal tumors, and mutations in epidermal growth factor receptor (EGFR) in non-small cell lung cancer (NSCLC). In the particular case of lung cancer, patients are screened for molecular alterations in EGFR, ALK, and ROS1 and for PD-L1 protein expression to optimize treatment outcomes. The identification of EGFR mutations as a key driver event in lung adenocarcinoma patients led to the development of small molecule tyrosine kinase inhibitors (TKIs); including first-generation (gefitinib [1, 2], erlotinib [3]), second-generation (afatinib [4]), and third-generation (osimertinib [5, 6], rociletinib (CO1686) [7]) inhibitors. Accordingly, the identification of driver mutations in EGFR that in turn were actionable alterations has had a significant impact on molecular profiling of biomarkerdriven targeted therapies in NSCLC, as well as in many other tumors $[8,9]$.

Currently, tissue biopsy is the gold standard for the determination of molecular alterations in lung cancer tumors. However, it has limitations. For example, most biopsies employ smallor fine-needle aspiration techniques that are often insufficient for genomic analysis after the initial histology diagnosis. The small size of the biopsy and cytology specimens also makes it impossible to determine the presence of residual disease by classical hematoxylin and eosin pathology and for accurate diagnosis at initial stages $[10,11]$. In addition, both inter- and intratumoral heterogeneity may limit the genotyping tests results, which can have a significant impact on treatment, particularly when the mechanisms for resistance to EGFR inhibitors need to be evaluated [12]. In this regard, EGFR mutant-positive lung adenocarcinoma has been associated with moderate-to-high intratumoral heterogeneity in all NSCLC subtypes, which necessitates the analysis of EGFR mutations in circulating tumor DNA due to its ability to overcome intratumoral heterogeneity [13-15]. Finally, it is worth mentioning the risks associated with biopsy in older lung cancer patients and patients with an inaccessible tumor location.

Accordingly, new techniques have been developed to study tumor biomarkers in liquid biopsies [16-18], such as mutation analysis in plasma-derived circulating cell-free DNA (cfDNA), which is released by tumor cells in the blood and which is considered the optimal candidate for clinical application [19-22]. In addition, cfDNA analysis can accurately determine tumor progression, diagnosis, and possible distant metastasis, it can predict prognosis as well as more effectively individualize the targeted therapy, and it is less invasive for the patient. Moreover, liquid biopsy is the only sample source that has been officially approved for clinical use in lung cancer patients $[16,17,23,24]$.

However, it is still difficult to preserve the integrity of the cfDNA population, particularly during plasma sample shipping. Blood collection procedures have to be carefully considered 
to maintain cfDNA integrity until its analysis, especially temperature and preservative conditions [25]. Accordingly, the main objective of this work was to analyze the concordance in cell- and DNA-stabilizing properties between cell-free DNA blood collection tubes (cfDNA BCTs) and the traditional shipping method, comparing the performance of the two methodologies in the detection of the EGFR mutational state. EGFR testing was performed using the cobas ${ }^{\circledR}$ EGFR Mutation Test v2 in all liquid biopsy samples. This technique is a realtime polymerase chain reaction (RT-PCR)-based test that can identify 42 different EGFR gene mutations in exons 18, 19, 20, and 21 of the gene; it has different detection limits for tissuederived DNA and plasma-derived cfDNA samples [26]. Moreover, a secondary objective was to provide a proof of concept of an alternative shipment method for these specific blood samples to an external laboratory as a real-life clinical practice experience.

\section{METHODS}

\section{Compliance with Ethics Guidelines}

The Independent Ethics Committee of Arnau Vilanova Hospital approved the study protocol, and all patients provided written informed consent before their inclusion, in addition to permission for the use of their blood samples (EDTA tubes and BCTs) in EGFR mutation analysis. The study was carried out in accordance with the Declaration of Helsinki and its amendments and with all applicable regulatory requirements.

\section{Sample Collection}

A total of 49 patients diagnosed with advanced NSCLC from three Spanish centers (Hospital del Doctor Peset, Hospital General de Castellon, and Hospital Arnau de Vilanova) participated in this study. The diagnosis was consistent with the clinical practice of the centers, with oncologists including patients meeting either of the following criteria: (a) absence of a tissue biopsy sample to analyze EGFR mutational status for a first-line treatment or due to high heterogeneity in NSCLC where tissue biopsy was negative, even though the clinical characteristics suggested, with high probability, the presence of EGFR mutant; and (b) patient treatment with TKIs for monitoring and analysis of progression.

Duplicate blood samples were obtained and shipped to Vall d'Hebron Hospital for processing and storage using two distinct methods in parallel: (1) via cell-free DNA blood collection tubes (BCTs) (Roche), shipped at room temperature; and (2) via standard $\mathrm{K}_{2}$ EDTA tubes containing plasma (obtained via double centrifugation of blood at $3000 \mathrm{rpm}$ within $20 \mathrm{~min}$ of blood extraction and frozen immediately at $-80^{\circ} \mathrm{C}$ ), shipped frozen on dry ice (shipments were made weekly). Samples were collected within a 9-month period.

\section{Sample Processing and DNA Extraction for Liquid Biopsy}

In total, 98 NSCLC liquid biopsy samples were included in the validation. BCTs were received at room temperature and were submitted to an initial centrifugation at $3000 \times g$ for $10 \mathrm{~min}$. Supernatants were collected and centrifuged at $16,000 \times g$ for $1 \mathrm{~min}$ to obtain the plasma; several $1.5-\mathrm{mL}$ aliquots were then made. Circulating cfDNA was then obtained via automated DNA extraction from the $1.5-\mathrm{mL}$ plasma samples using a QIAsymphony DSP Virus/Pathogen Kit (Qiagen) following the manufacturer's protocol on a QIAsymphony instrument. All samples were eluted in $85 \mu \mathrm{L}$ of elution buffer, $75 \mu \mathrm{L}$ of which was used in the mutation PCR protocol.

\section{EGFR Mutation Status Analysis in cfDNA}

Mutation analysis was performed by qPCR using a cobas ${ }^{\circledR}$ EGFR Mutation Test v2 kit (Roche). Briefly, $75 \mu \mathrm{L}$ of each DNA was used in each assay on a cobas ${ }^{\circledR} \mathrm{Z} 480$ analyzer. This kit detects 42 EGFR mutations (including G19A/S/C in exon 18, deletions and complex mutations in exon 19, S768I, T790 M, and exon 20 insertions, and L858R in exon 21). A standard curve created 
using the internal control Cp genomic DNA was used to calculate the DNA concentration and copy number, which is also represented as the semi-quantitative index of each EGFR mutant sample. For a sample to be classified as EGFR mutation-positive in this analysis, it had to contain at least one activating mutation (i.e., exon 19 deletion, L858R, G719x, or L861Q).

\section{Statistical Analysis}

Statistical analyses were performed with G-Stat version 2.0. The McNemar test was used to analysis the EGFR mutation concordance between matched samples.

\section{RESULTS}

\section{Concordance of EGFR Mutation Detection in cfDNA Between cfDNA BCTs and the Routine Plasma Shipping Procedure}

In total, 98 liquid biopsy samples were analyzed, corresponding to 49 patients from three different hospitals, with paired samples shipped according to the two different shipping methodologies: (1) standard frozen plasma and (2) cfDNA BCTs. After the plasma was received in the laboratory and the DNA was extracted, the EGFR mutation status was analyzed by qPCR using a $\operatorname{cobas}^{\circledR}$ EGFR Mutation Test v2 kit (Fig. 1). Of the 49 cases, 5 were classified as invalid by cobas ${ }^{\circledR}$ with one of the two shipping methodologies analyzed. The results of the analysis, presented in Fig. 2a, revealed an $85.7 \%$ concordance between the two techniques. The rate improved to $95.4 \%$ after the exclusion of the five invalid samples (Fig. 2b). Concordance was defined as EGFR mutation-positive or EGFR mutation-negative, and the mutation type was the same in all EGFR-mutated samples, as shown in Supplementary Table 1.

Considering the current methodology (frozen plasma) for sending samples as the gold standard, we evaluated the sensitivity and specificity of the cfDNA BCT shipment. The McNemar test showed that there was no significant difference between the two shipping methods $(P=0.157)$.

The global agreement between the two shipping methods was $95.4 \%$, with a sensitivity of $100 \%$ and specificity of $94.6 \%$ for the cfDNA BCTs. Further analysis of the cfDNA BCTs determined a positive predictive value of $81.8 \%$ and negative predictive value of $100 \%$ (Fig. 2b).

Finally, we analyzed the discordant results. As mentioned above, five samples were considered invalid by cobas ${ }^{\circledR}$ in one of the two shipping procedures. The EGFR mutation statuses of the two discordant results in plasma were



Fig. 1 Schematic representation of patients' samples and the results of the EGFR mutation analysis 
a



Concordance: $(33+9) / 49=85.7 \%$

b

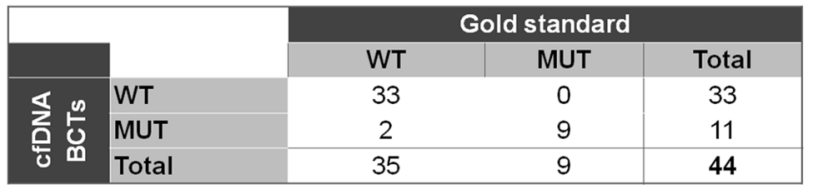

Concordance: $(33+9) / 44=95.4 \%$

Sensitivity: $[(T P /(T P+F N)]:[9 /(9+0)]=100 \%$

Specificity: $[(T N /(T N+F P)]:[35 /(35+2)]=\mathbf{9 4 . 6 \%}$

PPV: $[(T P /(T P+F P)]:[11 /(11+2)]=\mathbf{8 1 . 8 \%}$

NPV: $[(T N /(T N+F N)]:[35 /(35+0)]=100 \%$

Fig. 2 Comparison between cfDNA obtained from frozen plasma and BCT samples for determining EGFR mutation status. a All samples included in this study. b Comparative

compared with their corresponding results obtained via cobas ${ }^{\circledR}$ EGFR Mutation Test v1 kit analysis of formalin-fixed paraffin-embedded (FFPE) biopsy samples; the findings confirmed one of the results: an EGFR-del19 in the FFPE results was found to be EGFR-del19 in the cfDNA BCT results (Fig. 3).

\section{DISCUSSION}

Plasma cfDNA is emerging as a clinically relevant biomarker for the management and surveillance of cancer. Our results show that the analysis of the samples analyzed according to the two different shipping methods

pre-analytical workflow for liquid biopsy from blood collection and shipping to plasma preparation, such as for EGFR mutation status testing, is simpler for cfDNA BCTs than for standard $\mathrm{K}_{2}$ EDTA BCTs. For EGFR mutation analysis, cfDNA BCTs in liquid biopsy have the same sensitivity as the current methodology and very high specificity. Additionally, the semi-quantitative indices obtained by the two different workflow approaches show high concordance.

The methods used for whole blood processing, preservation, and storage are important considerations in circulating tumor DNA

\begin{tabular}{|c|c|c|c|}
\hline Patient & PCR Plasma & PCR BCTs & FFPE \\
\hline 1 & Invalid & WT & ---- \\
\hline 2 & Invalid & p.L858R & ---- \\
\hline 3 & WT & Invalid & --- \\
\hline 4 & Del 19 & Invalid & ---- \\
\hline 5 & WT & Invalid & --- \\
\hline 6 & WT & Del 19 & Del 19 \\
\hline 7 & WT & Del 19 & WT \\
\hline
\end{tabular}

Fig. 3 Comparison of EGFR mutation status between cfDNA and FFPE samples 
analysis $[25,27,28]$. Our results indicate that BCTs are preferable for the collection of circulating DNA in a clinical setting due to their favorable storage and shipping conditions. Other studies indicated that BCTs are preferable for the room-temperature preservation of cfDNA for up to 5 days and for preventing the release of genomic DNA, both in oncology and prenatal diagnostics [29-32].

Several pre-analytical steps are crucial to obtain good concordant mutation results between liquid biopsy and FFPE samples, beyond the natural behavior of the tumor, its shedding capacity and clinical stage (III or IV), the inter- and intratumoral heterogeneity, and tumor type. Some are related to the sample handling, including plasma extraction protocols, freeze/thaw cycles, sample shipment, and timing, which can affect the final mutation analysis of the EGFR gene in liquid biopsy.

We analyzed all discordant cases in duplicate to rule out the presence of artifacts. However, we did not include any additional methods to resolve discordant results because the protocol used to analyze the EGFR mutations was designed to consume all cfDNA obtained in each extraction. We thus had no additional cfDNA to perform further analysis with any high-sensitivity technique that might be recommended, such as droplet digital PCR or BEAMing. In line with ASCO-CAP recommendations [18], we compared the EGFR mutation results with those of some FFPE samples that had been previously analyzed in our laboratory with the cobas ${ }^{\circledR}$ EGFR Mutation Test v1, which is approved for mutation analysis in FFPE samples [33]. In this comparison, we only observed one EGFR mutant (Del19) in an FFPE sample, which was also detected by BCT analysis 2 months later.

In addition, our results are consistent with others showing that cfDNA BCTs can replace the standard collection and transport method due to their strong ability to detect tumorspecific mutations in key driver genes such as BRAF, KRAS, and PIK3CA in various solid cancers [34] and additional genes (EGFR and c-KIT) tested by BEAMing or Safe-SeqS procedures [35].

These cfDNA BCTs have some additional advantages in the collection and shipment processes: (1) they eliminate the need for onsite plasma processing and additional manipulation of samples by different individuals in the laboratories, reducing the variability in the pre-analytical steps and allowing standard roomtemperature shipping to the testing laboratory; (2) they provide a more standardized protocol that can be easily implemented in clinical laboratory routine; and (3) the process is less expensive. The price of EDTA tubes and dry ice special courier services is about $€ 78$ per sample, whereas BCTs and standard courier is about $€ 11$. Additionally, the dry ice service in this study has been established at once per week, which directly affects the EGFR test turnaround time.

This study has some limitations, such as a low number of samples included and a lack of extra cfDNA to perform additional experiments to elucidate the numbers of positive and negatives cases with both approaches.

\section{CONCLUSIONS}

The use of cfDNA BCTs for the EGFR mutation analysis in liquid biopsy presents the same sensitivity as the current methodology, and very high specificity. These cfDNA BCTs have additional advantages in the collection and further shipment processes. Therefore, due to their high performance, these tubes can be perfectly incorporated into the clinical routine for this EGFR mutational determination.

\section{ACKNOWLEDGEMENTS}

We thank the participants of the study.

Funding. This study was sponsored by Roche Farma S.A., Spain. The funder had no role in the study design, data collection and analysis, decision to publish, preparation of the manuscript, or payment of the charges related to article processing. All authors had full access to all of the data in this study and take complete responsibility for the integrity of the data and accuracy of the data analysis. Roche Farma S.A., Spain also funded the journal's Rapid Service Fee. 
Editorial Assistance. We thank Kevin Clayton for providing assistance with the English correction of the manuscript.

Authorship. All named authors met the International Committee of Medical Journal Editors (ICMJE) criteria for authorship for this article, take responsibility for the integrity of the work as a whole, and have given their approval for this version to be published.

Disclosures. Marta Sesé, Rosa Somoza, Inmaculada Maestu, Maria Martín Ureste, Alfredo Sanchez, Juan Felipe Cordoba, Irene Sansano, Griselda Venturas, Santiago Ramón y Cajal, and Javier Hernández-Losa have nothing to disclose.

Compliance with Ethics Guidelines. All procedures performed in studies involving human participants were in accordance with the ethical standards of the institutional and/or national research committee (Committee Hospital Arnau Vilanova CEIC-1796) and with the 1964 Helsinki declaration and its later amendments or comparable ethical standards. All patients provided written informed consent before their inclusion, in addition to permission for the use of their blood samples (EDTA tubes and BCTs) in EGFR mutation analysis.

Open Access. This article is distributed under the terms of the Creative Commons Attribution-NonCommercial 4.0 International License (http://creativecommons.org/licenses/ by-nc/4.0/), which permits any noncommercial use, distribution, and reproduction in any medium, provided you give appropriate credit to the original author(s) and the source, provide a link to the Creative Commons license, and indicate if changes were made.

\section{REFERENCES}

1. Mok TS, Wu YL, Thongprasert S, Yang CH, Chu DT, Saijo N, et al. Gefitinib or carboplatin-paclitaxel in pulmonary adenocarcinoma. $\mathrm{N}$ Engl J Med.
2009;361(10):947-57. https://doi.org/10.1056/ NEJMoa0810699.

2. Lynch TJ, Bell DW, Sordella R, Gurubhagavatula S, Okimoto RA, Brannigan BW, et al. Activating mutations in the epidermal growth factor receptor underlying responsiveness of non-small-cell lung cancer to gefitinib. $\mathrm{N}$ Engl $\mathrm{J}$ Med. 2004;350(21):2129-39. https://doi.org/10.1056/ NEJMoa040938.

3. Rosell R, Carcereny E, Gervais R, Vergnenegre A, Massuti B, Felip E, et al. Erlotinib versus standard chemotherapy as first-line treatment for European patients with advanced EGFR mutation-positive non-small-cell lung cancer (EURTAC): a multicentre, open-label, randomised phase 3 trial. Lancet Oncol. 2012;13(3):239-46. https://doi.org/10.1016/ S1470-2045(11)70393-X.

4. Yang JC, Wu YL, Schuler M, Sebastian M, Popat S, Yamamoto N, et al. Afatinib versus cisplatin-based chemotherapy for EGFR mutation-positive lung adenocarcinoma (LUX-Lung 3 and LUX-Lung 6): analysis of overall survival data from two randomised, phase 3 trials. Lancet Oncol. 2015;16(2):141-51. https://doi.org/10.1016/S14702045(14)71173-8.

5. Janne PA, Yang JC, Kim DW, Planchard D, Ohe Y, Ramalingam SS, et al. AZD9291 in EGFR inhibitorresistant non-small-cell lung cancer. N Engl J Med. 2015;372(18):1689-99. https://doi.org/10.1056/ NEJMoa1411817.

6. Gil-Bazo I, Rolfo C. AZD9291 in TKI EGFR resistance in non-small cell lung cancer and the new concept of phase I trials. Transl Lung Cancer Res. 2016;5(1):85-8. https://doi.org/10.3978/j.issn. 2218-6751.2015.07.02.

7. Sequist LV, Rolfe L, Allen AR. Rociletinib in EGFRmutated non-small-cell lung cancer. N Engl J Med. 2015;373(6):578-9. https://doi.org/10.1056/ NEJMc1506831.

8. Tan DS, Mok TS, Rebbeck TR. Cancer genomics: diversity and disparity across ethnicity and geography. J Clin Oncol. 2016;34(1):91-101. https://doi. org/10.1200/JCO.2015.62.0096.

9. Lee DH. Treatments for EGFR-mutant non-small cell lung cancer (NSCLC): the road to a success, paved with failures. Pharmacol Ther. 2017;174:1-21. https://doi.org/10.1016/j. pharmthera.2017.02.001.

10. Wang S, Yu B, Ng CC, Mercorella B, Selinger CI, O'Toole SA, et al. The suitability of small biopsy and cytology specimens for EGFR and other mutation testing in non-small cell lung cancer. Transl Lung 
Cancer Res. 2015;4(2):119-25. https://doi.org/10. 3978/j.issn.2218-6751.2015.01.05.

11. Ofiara LM, Navasakulpong A, Ezer N, Gonzalez AV. The importance of a satisfactory biopsy for the diagnosis of lung cancer in the era of personalized treatment. Curr Oncol. 2012;19(Suppl 1):S16-23. https://doi.org/10.3747/co.19.1062.

12. Piotrowska Z, Niederst MJ, Karlovich CA, Wakelee HA, Neal JW, Mino-Kenudson M, et al. Heterogeneity underlies the emergence of EGFRT790 wildtype clones following treatment of T790 M-positive cancers with a third-generation EGFR inhibitor. Cancer Discov. 2015;5(7):713-22. https://doi.org/ 10.1158/2159-8290.CD-15-0399.

13. Zhang Y, Chang L, Yang Y, Fang W, Guan Y, Wu A, et al. Intratumor heterogeneity comparison among different subtypes of non-small-cell lung cancer through multi-region tissue and matched ctDNA sequencing. Mol Cancer. 2019;18(1):7. https://doi. org/10.1186/s12943-019-0939-9.

14. Wan R, Wang Z, Lee JJ, Wang S, Li Q, Tang F, et al. Comprehensive analysis of the discordance of EGFR mutation status between tumor tissues and matched circulating tumor DNA in advanced non-small cell lung cancer. J Thorac Oncol. 2017;12(9):1376-87. https://doi.org/10.1016/j.jtho. 2017.05.011.

15. Jing CW, Wang Z, Cao HX, Ma R, Wu JZ. High resolution melting analysis for epidermal growth factor receptor mutations in formalin-fixed paraffin-embedded tissue and plasma free DNA from non-small cell lung cancer patients. Asian Pac J Cancer Prev. 2014;14(11):6619-23.

16. Crowley E, Di Nicolantonio F, Loupakis F, Bardelli A. Liquid biopsy: monitoring cancer-genetics in the blood. Nat Rev Clin Oncol. 2013;10(8):472-84. https://doi.org/10.1038/nrclinonc.2013.110.

17. Pisapia P, Malapelle U, Troncone G. Liquid biopsy and lung cancer. Acta Cytol. 2018;5:1-8. https:// doi.org/10.1159/000492710.

18. Merker JD, Oxnard GR, Compton C, Diehn M, Hurley P, Lazar AJ, et al. Circulating tumor DNA analysis in patients with cancer: American Society of Clinical Oncology and College of American Pathologists Joint Review. J Clin Oncol. 2018;36(16):1631-41. https://doi.org/10.1200/JCO. 2017.76.8671.

19. Alix-Panabieres C, Pantel K. Clinical applications of circulating tumor cells and circulating tumor DNA as liquid biopsy. Cancer Discov. 2016;6(5):479-91. https://doi.org/10.1158/2159-8290.CD-15-1483.
20. Diaz LA Jr, Bardelli A. Liquid biopsies: genotyping circulating tumor DNA. J Clin Oncol. 2014;32(6):579-86. https://doi.org/10.1200/JCO. 2012.45.2011.

21. Heitzer E, Ulz P, Geigl JB. Circulating tumor DNA as a liquid biopsy for cancer. Clin Chem. 2015;61(1):112-23. https://doi.org/10.1373/ clinchem.2014.222679.

22. Luo W, Rao M, Qu J, Luo D. Applications of liquid biopsy in lung cancer-diagnosis, prognosis prediction, and disease monitoring. Am J Transl Res. 2018;10(12):3911-23.

23. Sacher AG, Komatsubara KM, Oxnard GR. Application of plasma genotyping technologies in nonsmall cell lung cancer: a practical review. J Thorac Oncol. 2017;12(9):1344-56. https://doi.org/10. 1016/j.jtho.2017.05.022.

24. Lim M, Kim CJ, Sunkara V, Kim MH, Cho YK. Liquid biopsy in lung cancer: clinical applications of circulating biomarkers (CTCs and ctDNA). Micromachines (Basel). 2018;9(3):E100. https://doi.org/ 10.3390/mi9030100.

25. Parpart-Li S, Bartlett B, Popoli M, Adleff V, Tucker L, Steinberg $R$, et al. The effect of preservative and temperature on the analysis of circulating tumor DNA. Clin Cancer Res. 2017;23(10):2471-7. https:// doi.org/10.1158/1078-0432.CCR-16-1691.

26. Malapelle U, Sirera R, Jantus-Lewintre E, Reclusa P, Calabuig-Farinas S, Blasco A, et al. Profile of the Roche cobas(R) EGFR mutation test v2 for nonsmall cell lung cancer. Expert Rev Mol Diagn. 2017;17(3):209-15. https://doi.org/10.1080/ 14737159.2017.1288568.

27. Grolz D, Hauch S, Schlumpberger M, Guenther K, Voss T, Sprenger-Haussels M, et al. Liquid biopsy preservation solutions for standardized pre-analytical workflows-venous whole blood and plasma. Curr Pathobiol Rep. 2018;6(4):275-86. https://doi. org/10.1007/s40139-018-0180-z.

28. Risberg B, Tsui DWY, Biggs H, Ruiz-Valdepenas Martin de Almagro A, Dawson SJ, Hodgkin C et al. Effects of collection and processing procedures on plasma circulating cell-free DNA from cancer patients. J Mol Diagn. 2018;20(6):883-92. https:// doi.org/10.1016/j.jmoldx.2018.07.005.

29. Norton SE, Luna KK, Lechner JM, Qin J, Fernando MR. A new blood collection device minimizes cellular DNA release during sample storage and shipping when compared to a standard device. J Clin Lab Anal. 2013;27(4):305-11. https://doi.org/10. 1002/jcla.21603. 
30. Norton SE, Lechner JM, Williams T, Fernando MR. A stabilizing reagent prevents cell-free DNA contamination by cellular DNA in plasma during blood sample storage and shipping as determined by digital PCR. Clin Biochem. 2013;46(15):1561-5. https://doi.org/10.1016/j.clinbiochem.2013.06. 002.

31. Fernando MR, Chen K, Norton S, Krzyzanowski G, Bourne D, Hunsley B, et al. A new methodology to preserve the original proportion and integrity of cell-free fetal DNA in maternal plasma during sample processing and storage. Prenat Diagn. 2010;30(5):418-24. https://doi.org/10.1002/pd. 2484 .

32. Warton K, Yuwono NL, Cowley MJ, McCabe MJ, So A, Ford CE. Evaluation of Streck BCT and PAXgene stabilised blood collection tubes for cell-free circulating DNA studies in plasma. Mol Diagn Ther. 2017;21(5):563-70. s40291-017-0284-X.
33. Benlloch S, Botero ML, Beltran-Alamillo J, Mayo C, Gimenez-Capitan A, de Aguirre I, et al. Clinical validation of a PCR assay for the detection of EGFR mutations in non-small-cell lung cancer: retrospective testing of specimens from the EURTAC trial. PLoS One. 2014;9(2):e89518. https://doi.org/ 10.1371/journal.pone.0089518.

34. Ahlborn LB, Madsen M, Jonson L, Nielsen FC, Lassen $\mathrm{U}$, Yde CW, et al. Concordance of mutation detection in circulating tumor DNA in early clinical trials using different blood collection protocols. Clin Lab. 2017;63(10):1755-9. https://doi.org/10. 7754/Clin.Lab.2017.170516.

35. Medina Diaz I, Nocon A, Mehnert DH, Fredebohm J, Diehl F, Holtrup F. Performance of Streck cfDNA blood collection tubes for liquid biopsy testing. PLoS One. 2016;11(11):e0166354. https://doi.org/ 10.1371/journal.pone.0166354. 